\title{
A Swarm Intelligence Graph-Based Pathfinding Algorithm Based on Fuzzy Logic (SIGPAF): A Case Study on Unmanned Surface Vehicle Multi-Objective Path Planning
}

\author{
Charis Ntakolia ${ }^{1,2, *(D)}$ and Dimitrios V. Lyridis ${ }^{1}$ (D) \\ 1 Laboratory for Maritime Transport, National Technical University of Athens, 15773 Athens, Greece; \\ dsvlr@mail.ntua.gr \\ 2 Machining Technology and Production Management, Sector of Materials Engineering, Department of \\ Aeronautical Studies, Hellenic Air Force Academy, 13672 Tatoi, Greece \\ * Correspondence: cntakolia@naval.ntua.gr or charis.ntakolia@hafahaf.onmicrosoft.com
}

check for

updates

Citation: Ntakolia, C.; Lyridis, D.V. A Swarm Intelligence Graph-Based Pathfinding Algorithm Based on Fuzzy Logic (SIGPAF): A Case Study on Unmanned Surface Vehicle Multi-Objective Path Planning. J. Mar. Sci. Eng. 2021, 9, 1243. https:// doi.org/10.3390/jmse9111243

Academic Editors: Sergei Chernyi and Marco Cococcioni

Received: 29 September 2021 Accepted: 7 November 2021 Published: 9 November 2021

Publisher's Note: MDPI stays neutral with regard to jurisdictional claims in published maps and institutional affiliations.

Copyright: (c) 2021 by the authors. Licensee MDPI, Basel, Switzerland. This article is an open access article distributed under the terms and conditions of the Creative Commons Attribution (CC BY) license (https:// creativecommons.org/licenses/by/ $4.0 /)$.

\begin{abstract}
Advances in robotic motion and computer vision have contributed to the increased use of automated and unmanned vehicles in complex and dynamic environments for various applications. Unmanned surface vehicles (USVs) have attracted a lot of attention from scientists to consolidate the wide use of USVs in maritime transportation. However, most of the traditional path planning approaches include single-objective approaches that mainly find the shortest path. Dynamic and complex environments impose the need for multi-objective path planning where an optimal path should be found to satisfy contradicting objective terms. To this end, a swarm intelligence graphbased pathfinding algorithm (SIGPA) has been proposed in the recent literature. This study aims to enhance the performance of SIGPA algorithm by integrating fuzzy logic in order to cope with the multiple objectives and generate quality solutions. A comparative evaluation is conducted among SIGPA and the two most popular fuzzy inference systems, Mamdani (SIGPAF-M) and TakagiSugeno-Kang (SIGPAF-TSK). The results showed that depending on the needs of the application, each methodology can contribute respectively. SIGPA remains a reliable approach for real-time applications due to low computational effort; SIGPAF-M generates better paths; and SIGPAF-TSK reaches a better trade-off among solution quality and computation time.
\end{abstract}

Keywords: path planning; metaheuristic algorithms; unmanned surface vehicles; maritime transportation; optimization; fuzzy logic

\section{Introduction}

The wide use of automated robotics and vehicles in various applications emerged the need for autonomous path planning strategies that remove the human presence from the decision-making [1-3]. Typical path planning strategies rely on single-objective models, such as minimization of traveled distance, time, or energy consumption, among others, where traditional and popular optimization algorithms are employed for finding an optimal solution. For instance, the Ant Colony Optimization (ACO) algorithm [4,5] or graph-based pathfinding algorithms such as Dijkstra [6] and $A^{*}[7,8]$ are commonly used for solving single-objective path planning problems, even in maritime applications, for unmanned surface vehicle (USV) path planning [9].

However, trends in robotic motion and computer vision allow autonomous robotic vehicles to move in complex and dynamic environments. To this end, new approaches have been developed to cope with multi-objective path planning problems where an equilibrium among conflicting objectives/criteria should be found [10]. Conventional approaches for addressing multi-objective (MO) path planning problems include the weighted sum method (WSM) or Pareto optimality. However, the WSM approach combines the objectives into one single-objective scalar function in an attempt to generate an efficient solution for 
the MO problem under consideration. Unfortunately, the selection of the weights leads to different results with the risk of trapping to local optimal solutions. On the other hand, the Pareto optimality approach overcomes the limitations of WSM by providing various solutions to the decision maker. This constitutes the Pareto optimality approach among the most popular for solving multi-objective optimization problems, especially with conflicting objectives. In Pareto optimality approaches, only one 'compromise' Pareto solution is the optimal/preferable one, making the presence of a decision maker imperative during the process-a limitation that is crucial in real-time applications of automated vehicles [11-14]. Fuzzy logic has also been integrated to optimization algorithms for solving multi-objective path planning problems. This approach enables the automated decision making of an optimal path based on fuzzy rules. These approaches employ mainly the conventional fuzzy control systems, such as the Mamdani fuzzy control and Takagi-Sugeno-Kang fuzzy (TSK) control [15-17]. However, algorithms combined with fuzzy logic present the weakness of high computational expense [18].

Even if significant work has been done on autonomous underwater vehicles [15,16], few studies have been focused on using fuzzy logic for solving multi-objective path planning problems for USVs. In the context of USV path planning, various methodologies have been developed for path planning based on heuristics. Most of these approaches address the single-objective path planning by finding the shortest path for global path planning enriched with obstacle avoidance in case of local path planning. Specifically, methodologies have been proposed for finding the shortest path based on popular metaheuristics that are commonly used for addressing the Traveling Salesman Problem (TSP). These approaches include improvements to the Ant Colony Optimization (ACO) algorithm [19] by dynamically selecting the pheromone volatility coefficient to enhance the searching ability of the algorithm; Genetic Algorithm (GA) by using the multidomain inversion to increase the offspring and consecutively increase the convergence speed [20], and in cooperation with other heuristic algorithms for task assignment and obstacle avoidance in the case of swarm of USVs [21]; $\mathrm{A}^{*}$ algorithm for safer routes by adding a smoothing process [8], or for obstacle avoidance [7,22] combined in a hybrid scheme with Artificial Potential Field (APF) algorithm [23]; rapidly-exploring random tree (RRT) algorithm with adaptive hybrid dynamic step size and target attractive force [24]; and Particle Swarm Optimization (PSO) [25] with orientation-angle-based grouping for higher convergence speed [26]. Other approaches are based on B-splines for collision avoidance where the optimal path is generated in multiple steps so that an initial shortest path will be smoothed and directed away from obstacles [27]. In [28], a fuzzy decision-making process was combined with the APF algorithm for obstacle avoidance in USV path planning.

Regarding multi-objective path planning in case of USV, a multi-objective, nonlinear optimization model was formulated for path planning of USV with currents effects. Most of these approaches are based on WSM and Pareto Optimality. Regarding WSM, an improvement of the $\mathrm{A}^{*}$ algorithm is proposed for minimizing the distance voyage time and energy consumption of the USV as a single objective [29]. To address the USV multi-objective problem, the dynamic augmented multi-objective particle swarm optimization algorithm was proposed based on Pareto optimal path set [30]. A* algorithm was used in [7] for optimal path planning of USV with dynamic obstacles and ocean currents. A hybrid metaheuristic based on quantum computing and ACO was proposed in [31] that is able to find an optimal path with multiple objectives considered as a single weighted sum function. A similar approach was proposed in [32] with quantum computing and PSO for local path planning and obstacle avoidance. Another study that proposed a modification of PSO for solving the global multi-objective path planning problem was presented in [33]. The problem was formulated as a TSP problem with a weighted sum objective function of multiple objectives and the PSO algorithm was combined with chaotic maps to increase the search velocity of the solution space.

As we mentioned above, the methodologies presented in the related literature are limited to traditional optimization methodologies, focusing on minimizing over one objective 
using mainly the WSM, or in some cases, the Pareto optimality for multi-objective path planning problems. Moreover, path-search approaches, such as Dijkstra and $\mathrm{A}^{*}$, demand high computational effort, especially in cases of complex and dynamic environments with enlarged maps [34,35]. To this end, in order to cope with the aforementioned limitations, an improved swarm intelligence graph-based pathfinding algorithm enhanced with fuzzy logic (SIGPAF) is proposed for addressing multi-objective path planning problems in the case of USVs. The proposed algorithm is based on the state-of-the-art algorithm SIGPA [11] that has been tested and used successfully in solving multi-objective graph-based path planning problems in real time. The aims of this study are as follows:

- Enhance the ability of the SIGPA algorithm to generate an optimal path among multiple objectives by integrating fuzzy logic and fuzzy rules;

- Perform a comparative evaluation among the two most popular fuzzy inference systems, such as Mamdani [36] and Takagi-Sugeno-Kang [37], and the original SIGPA algorithm to examine the contribution of the integration of fuzzy logic to the algorithm's performance;

- Evaluate the effectiveness of the proposed algorithms to solve multi-objective path planning problems in the case of USVs.

The proposed fuzzy approaches of the original SIGPA algorithm have also been developed in the frame of the OPTINET project that has been co-financed by the European Regional Development Fund of the European Union and Greek national funds through the operational program "Competitiveness, Entrepreneurship and Innovation", under the call "Research-Create-Innovate" (project code: T1E $\Delta$ K-01907).

\section{Materials and Methods}

\subsection{Problem Definition and Formulation}

This study aims to investigate the graph-based multi-objective path planning problems. Specifically, the path planning problem for USVs with the appearance of static obstacles is addressed in the context of the evaluation of the developed metaheuristic algorithm (FTSI). The study focuses on finding the optimal path for a USV from an initial node to multiple targets that should be visited by minimizing the traveled distance, the path deviations with respect to the angle of the turn that the USV should perform, and the energy consumption due to sea currents. The aforementioned objective terms, or optimization criteria, are modeled as follows:

Objective term 1: Traveled distance

$$
\min D=\sum_{n \in \mathcal{N}} \sum_{\substack{m \in \mathcal{N}: \\(n, m) \in \varepsilon}} d_{n m}=\sum_{n \in \mathcal{N}} \sum_{\substack{m \in \mathcal{N}: \\(n, m) \in \varepsilon}}\left(\sqrt{\left(m_{x}-n_{x}\right)^{2}+\left(m_{y}-n_{y}\right)^{2}}\right)
$$

where $\mathcal{N}$ and $\varepsilon$ are the set of nodes that form the graph and the set of edges of the graph, respectively; $d_{n m}$ is the distance metric used to calculate the distance between the nodes $n$ and $m$. In our study, the Euclidean distance was used. The variables $n_{x}, m_{x}$ and $n_{y}, m_{y}$ are the geographical coordinates of nodes $n$ and $m$ on the horizontal and vertical axes, respectively. This objective term minimizes the traveled distance of the USV in order to reach the target from each initial position.

Objective term 2: Path deviations

$$
\min \varphi=\sum_{l \in \mathcal{N}} \sum_{\substack{m \in \mathcal{N}: \\(l, m) \in \mathcal{E}}} \sum_{\substack{n \in \mathcal{N}: \\(m, n) \in \mathcal{E}}} \varphi_{l m n}
$$

where $\varphi_{l m n}$ is the angle that is formed by the consecutive edges $(l, m)$ and $(m, n)$. This objective term minimizes the brut changes along the path so the route will be as smooth as possible. 
Objective term 3: Energy consumption

$$
\min E C=\sum_{m \in \mathcal{N}} \sum_{\substack{n \in \mathcal{N}: \\(m, n) \in \mathcal{\varepsilon}}} \frac{d_{m n}}{\left|\vec{V}+\vec{v}_{c}\right|} F
$$

where $F$ is the fuel consumption per unit time $(\mathrm{kg} / \mathrm{h}), V$ is the velocity of the USV, and $v_{c}$ is the velocity of the currents. This term minimizes the energy consumption of the USV along the path. To this end, the term forces the USV to move in accordance with the direction of the currents so less energy will be needed for the USV to perform the route. Based on the literature, if a USV is moving against the currents, more energy is needed to retain a certain velocity during a route $[30,31,38,39]$.

\subsection{Fuzzy Swarm Intelligence Graph-Based Pathfinding Algorithm (SIGPAF)}

In this study, a novel, swarm intelligence graph-based pathfinding algorithm based on fuzzy logic is proposed for addressing multi-objective graph-based problems. The algorithm is inspired and based on the main principles of $\mathrm{A}^{*}$ algorithm and the state-of-the-art metaheuristic algorithm SIGPA [11] that is used to solve multi-objective path planning problems.

\subsubsection{SIGPA Algorithm}

SIGPA algorithm was developed to address the limitations of the $\mathrm{A}^{*}$ algorithm in solving practical path planning problems, such as its space complexity and computational demands $[40,41]$ for solving multi-objective path planning problems that are formulated in graphs. The main operation of the SIGPA algorithm is based on a greedy path search approach and on a root-mean-square error metric for identifying the 'best' neighbor at each step. It solves the Traveling Salesman Problem (TSP), where multiple points of interest (POI) should be visited; then, it proposes an optimal path to visit the selected POIs in order. Specifically, the SIGPA algorithm consists of 3 processes: (i) the POI selection ranking system (POI-SRS) that selects the POI visiting sequence; (ii) the greedy graph-based pathfinding algorithm that computes a feasible path among two POIs; and (iii) the swarm process [11].

Given an initial solution, the process is performed in parallel for all particles of the swarm and is repeated until the termination criteria are met, such as the maximum number of iterations. Each particle searches for a candidate solution with the greedy graph-based pathfinding algorithm, called GPA. This enables the algorithm to avoid stationary points and probably lead a better solution. Let $\mathcal{U}_{n}$ be the set of the neighbor nodes that are connected with arcs with node $n ; \mathcal{N}$ be the queue list with the neighbor nodes to be checked; the $\mathcal{P}$ queue list with the point of interest (POI) nodes to be visited; the route list $\mathcal{R}$ with the visited node sequence; the starting node $s$; and the ending node $e$. Let $\mathcal{N}$ be the route nodes that are not yet selected from the current solution $s_{c}, \mathcal{U}_{n}$ be the neighbor nodes that are connected with arcs with node $n, \mathcal{P}$ be the selected POIs, $\mathcal{P}_{m}$ be the selected POIs that should be visited by each particle-node $m$, and $\mathcal{S}_{m}$ be the search space of feasible solutions of $m$. Let start and end be starting and ending nodes, $k$ be the number of population —nodes to be randomly selected from the current route solution, $\mathfrak{W}$ be the maximum number of iterations, $p$ be the acceptance probability of a solution, $k_{i}$ be the number of population after excluding infeasible solutions in $i^{\text {th }}$ iteration, iter be the current iteration, and tol be the acceptable error for convergence. Each path is referred to as a particle [11]. Figures 1 and 2 illustrate the flowcharts of GPA and SIGPA algorithms, respectively. 


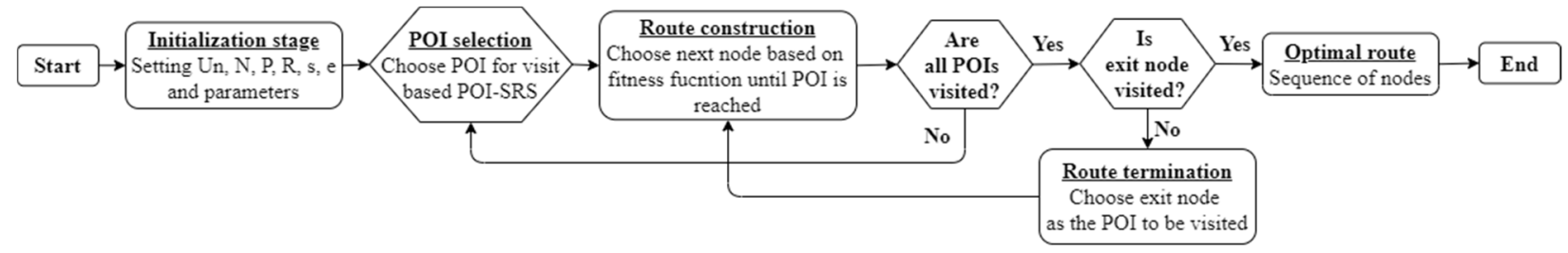

Figure 1. Flowchart of the greedy graph-based pathfinding algorithm (GPA) [11].

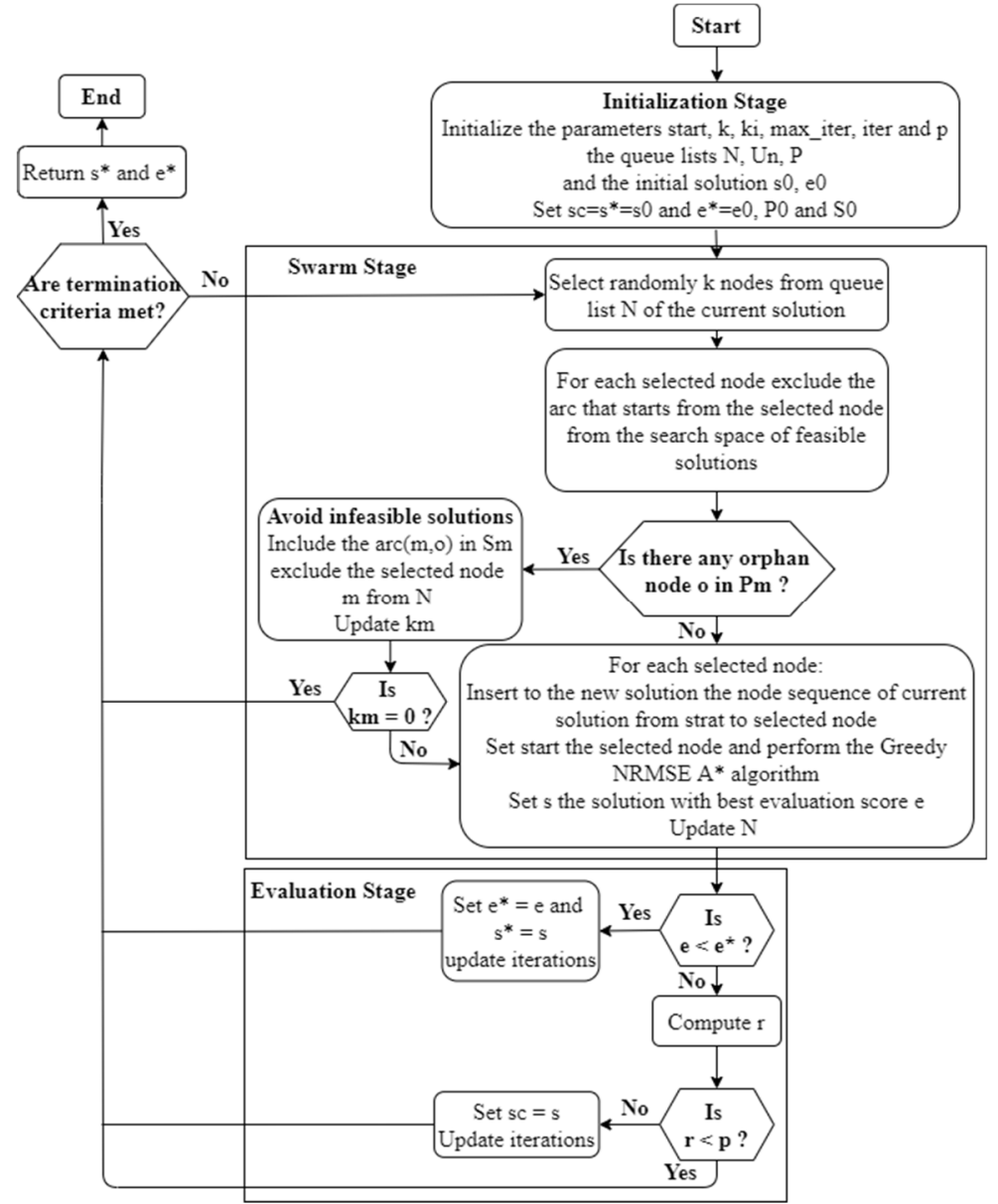

Figure 2. Flowchart of the SIGPA algorithm [11].

\subsubsection{Fuzzy Evaluation}

Fuzzy logic is employed to evaluate each node during the search of a path by a particle. Further, the same evaluation process is performed for the ranking of the retrieved paths among the population.

In our study, three objective terms are taken into account for the evaluation of a path, as presented in Section 2.1: (i) path distance (Equation (1)); (ii) path deviations (Equation (2)); (iii) energy consumption (Equation (3)). Each one is normalized and then fuzzified based on the membership functions shown in Figures 2-5. To this end, three fuzzy subsets are used for the input variables, while five were used for the output variable. Given each crisp value, the uncertainty can be modeled by fuzzy sets:

$$
\begin{gathered}
\widetilde{c}_{1, v}=\left\{x, \mu_{c_{1, v}}(x) \mid x \in U_{c_{1}}\right\}, v=\{\text { short, moderate, long }\} \\
\widetilde{c}_{2, v}=\left\{x, \mu_{c_{2, v},}(x) \mid x \in U_{c_{2}}\right\}, v=\{\text { smooth, adequate, brut }\} \\
\widetilde{c}_{3, v}=\left\{x, \mu_{c_{3, v}}(x) \mid x \in U_{c_{3}}\right\}, v=\{\text { low, medium, high }\}
\end{gathered}
$$


$\tilde{p}_{v}=\left\{x, \mu_{p_{v}},(x) \mid x \in U_{p}\right\}, v=\{$ very low, low, medium, high, very high $\}$

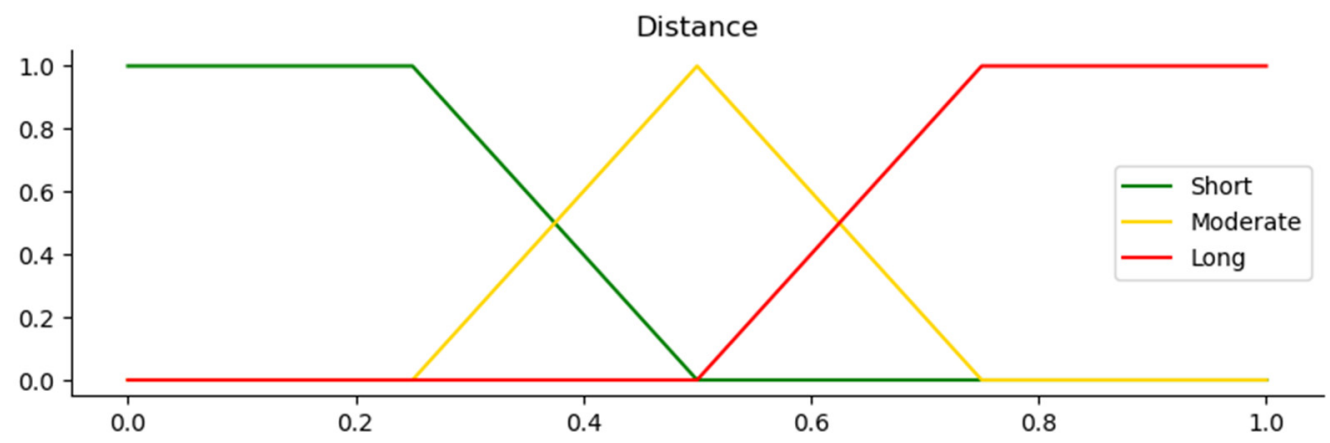

Figure 3. Membership function of Criterion 1 (Equation (1)) of traveled distance.

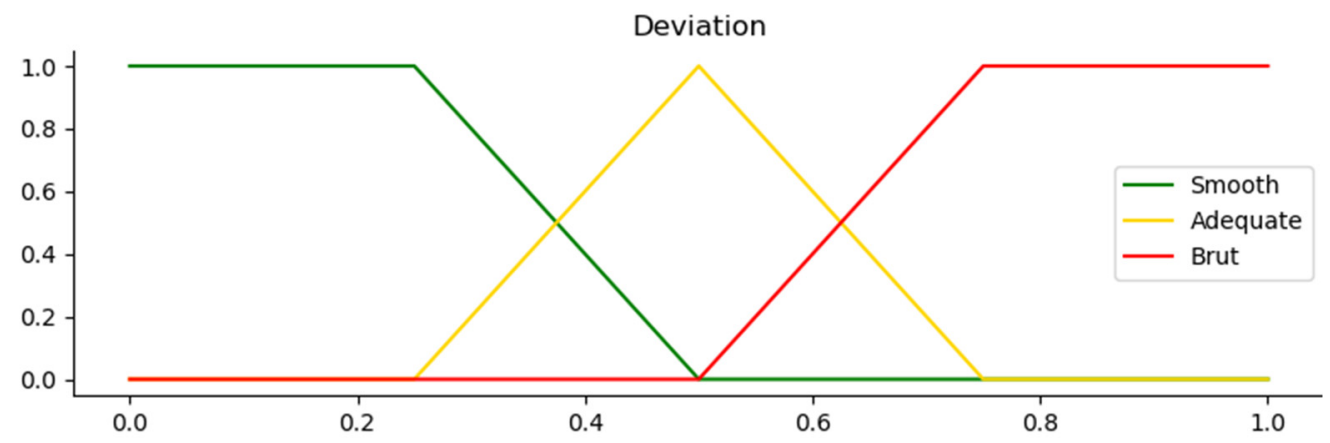

Figure 4. Membership function of Criterion 2 (Equation (2)) of path deviations.

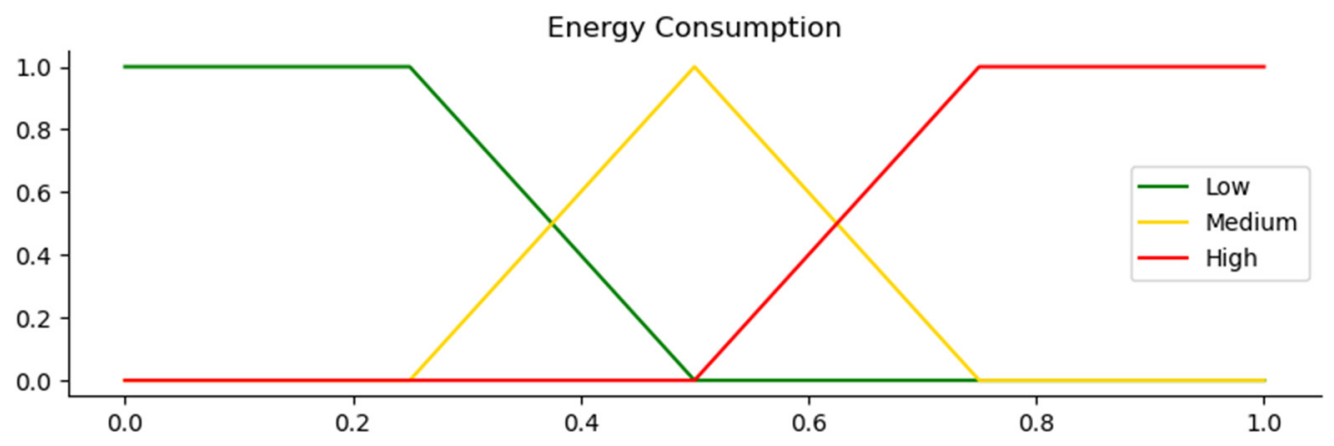

Figure 5. Membership function of Criterion 3 (Equation (3)) of energy consumption.

These fuzzy sets represent overlapping value intervals that can be expressed linguistically-e.g., "short", "moderate", "long" in the case of the path's distance $\left(c_{1}\right)$; "smooth", "adequate", and "brut" in case of path's deviations $\left(c_{2}\right)$; and "low", "medium", "high" in the case of the energy consumption of the USV in order to realize the path $\left(c_{3}\right)$. For this, three fuzzy universes are defined- $-U_{c_{1}}, U_{c_{2}}$, and $U_{c_{3}}$-representing the universe of discourse for the distance, deviations, and energy consumption, respectively. The universe $U_{p}$ corresponds to the quality of a path.

Similarly, the path's quality is determined by using the fuzzy rules defined in Table 1 and a Fuzzy Inference System. In our case, the Mamdani and Takagi-Sugeno-Kang (TSK) fuzzy controls are employed for a comparative evaluation. The quality of each path is then defuzzified into a crisp value (Figure 6). Figure 7 illustrates the flowchart of SIGPAF with the integration of a FIS in the SIGPA algorithm in the evaluation stages. 
Table 1. The fuzzy rules adopted for the FIS.

\begin{tabular}{|c|c|c|c|c|}
\hline Fuzzy Rules & Distance & Deviation & Energy Consumption & Path Quality \\
\hline Rule 1 & Short & Smooth & Low & Very High \\
\hline Rule 2 & Short & Smooth & Medium & Very High \\
\hline Rule 3 & Short & Adequate & Low & Very High \\
\hline Rule 4 & Moderate & Smooth & Low & Very High \\
\hline Rule 5 & Short & Smooth & High & High \\
\hline Rule 6 & Short & Adequate & Medium & High \\
\hline Rule 7 & Short & Brut & Low & High \\
\hline Rule 8 & Moderate & Smooth & Medium & High \\
\hline Rule 9 & Moderate & Adequate & Low & High \\
\hline Rule 10 & Long & Smooth & Low & High \\
\hline Rule 11 & Short & Adequate & High & Medium \\
\hline Rule 12 & Short & Brut & Medium & Medium \\
\hline Rule 13 & Short & Brut & High & Medium \\
\hline Rule 14 & Moderate & Smooth & High & Medium \\
\hline Rule 15 & Moderate & Adequate & Medium & Medium \\
\hline Rule 16 & Moderate & Brut & Low & Medium \\
\hline Rule 17 & Long & Smooth & Medium & Medium \\
\hline Rule 18 & Long & Smooth & High & Medium \\
\hline Rule 19 & Long & Adequate & Low & Medium \\
\hline Rule 20 & Long & Brut & Low & Medium \\
\hline Rule 21 & Moderate & Adequate & High & Low \\
\hline Rule 22 & Moderate & Brut & Medium & Low \\
\hline Rule 23 & Moderate & Brut & High & Low \\
\hline Rule 24 & Long & Adequate & Medium & Low \\
\hline Rule 25 & Long & Adequate & High & Low \\
\hline Rule 26 & Long & Brut & Medium & Low \\
\hline Rule 27 & Long & Brut & High & Very Low \\
\hline
\end{tabular}

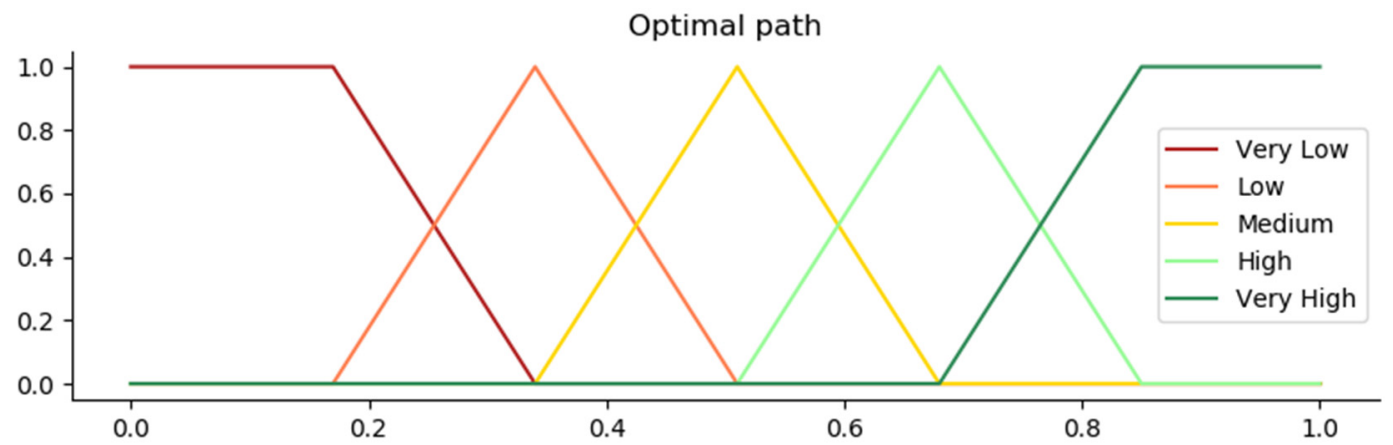

Figure 6. Membership function of the overall quality of the path.

The main advantage of Mamdani FIS is that the output can be presented both linguistically and by using a crisp value due to the implementation of the defuzzification process with a fuzzy set. This constitutes the Mamdani FIS as an effective approach to decision making systems [36]. TSK FIS, on the other hand, gives a crisp output value obtained by a weighted average of the rules' consequent [37], resulting a less computationally demanding approach compared with Mamdani. Therefore, these two types of FISs can be considered suitable for real-time applications of USV path planning and, in this study, the effectiveness of each one in a hybrid scheme with SIGPA metaheuristic is examined. 


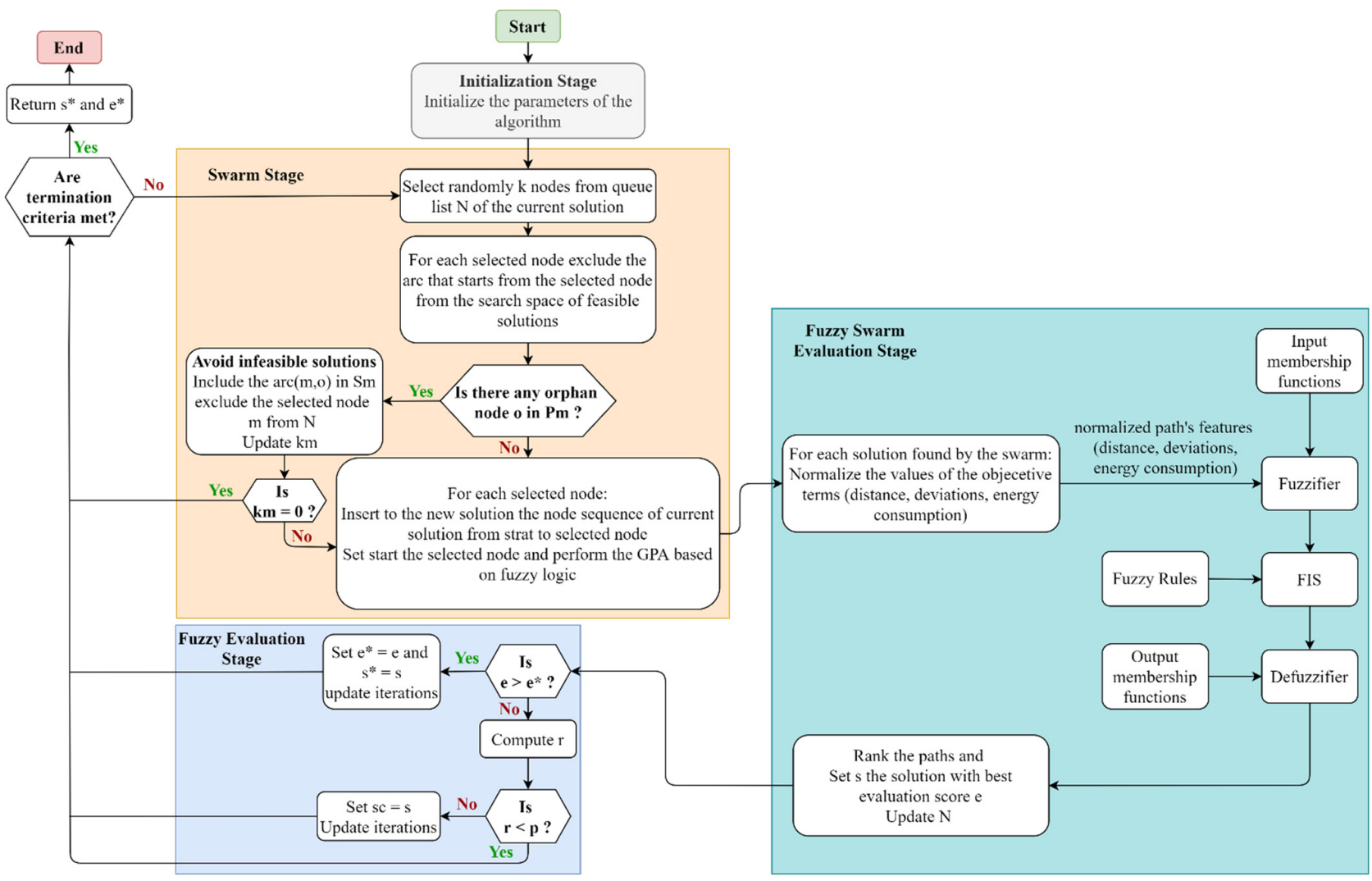

Figure 7. Flowchart of the SIGPAF algorithm.

\section{Results}

\subsection{Evaluation Methodology}

To evaluate the effectiveness of the proposed algorithm, a comparative evaluation among SIGPAF with Mamdani (SIGPAF-M), SIGPAF with Takagi-Sugeno-Kang (SIGPAFTSK), and SIGPA algorithm was conducted. Figure 8 illustrates the evaluation environment that is used for addressing the USV path planning problem described in Section 2.1. The grid area forms the graph that is used for path planning in the port area of Piraeus. In this area, the USV should perform specific missions that are defined for these case studies in order to visit selected targets among its path. The arcs that connect the nodes are shown in black lines within the grid area. The green node represents the initial node and the red ones the targets. For the evaluation, three missions are developed (Figure 9). In the first mission, the USV should travel from the initial position to a final target. The second mission consists of four targets, and the third mission of eight. The velocity and the direction of the currents were randomly assigned per node in each iteration. The current's velocity was set among 1.5 and $2.5 \mathrm{~m} / \mathrm{s}$ with clockwise and anticlockwise directions.

To evaluate the performance of the comparative algorithms under more realistic conditions - 10 scenarios of randomly generated graphs of 160 nodes, from 5 to 25 targets, and 356 permitted arcs. For each arch, a random current velocity was set from 1.5 and $2.5 \mathrm{~m} / \mathrm{s}$ with a clockwise or anticlockwise direction. Static objects, such as islands where randomly placed in the graphs (1 to 10). Each island was represented with different sizes by excluding 1 to 4 nodes from the graph. 


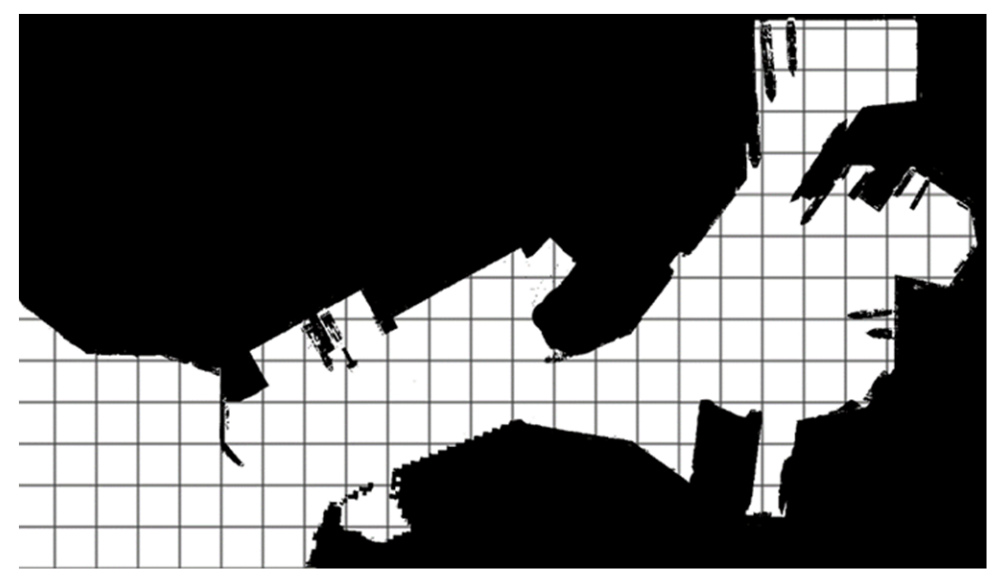

Figure 8. Simulation environment used for the comparative evaluation.
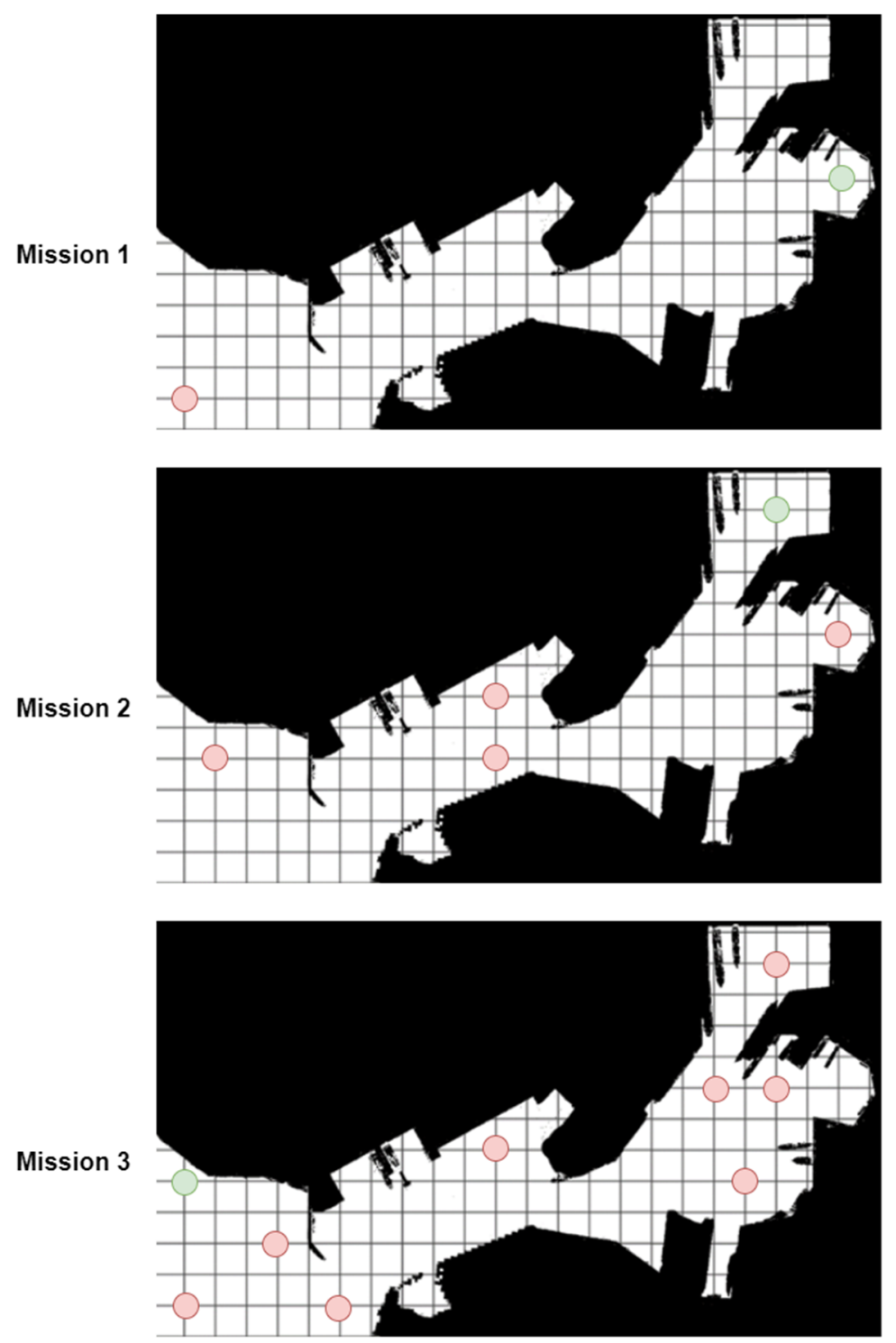

Figure 9. Missions used for the comparative evaluation. From top to bottom the Mission 1, Mission 2 and Mission 3 are illustrated, respectively. 
The case studies were designed based on the evaluation methodology adopted in similar studies in the literature $[8,11,42,43]$. They were simulated using Java, on Microsoft Windows 10 Environment as operational system, with an AMD Ryzen 7 3800X 8-Core Processor at $3.89 \mathrm{GHz}$ and 32GB RAM. The SIGPA algorithm was given by [11].

\subsection{Results}

Table 2 shows the mean computation time needed for each algorithm to find an optimal path. Table 3 shows the quality of the generated paths of each algorithm per mission with respect to the objective terms, such as traveled distance, energy consumption, and path deviations. Last Table 4 presents the experimental results of 10 random generated scenarios of SIGPA, SIGPAF-M, and SIGPAF-TSK.

Table 2. Mean Computing time in milliseconds (ms) of SIGPA, SIGPAF-M, and SIGPAF-TSK algorithms.

\begin{tabular}{ccccc}
\hline Algorithm & Mission 1 & Mission 2 & Mission 3 & Average \\
\hline SIGPA & 0.042 & $\mathbf{0 . 0 7 8}$ & $\mathbf{0 . 0 9 9}$ & $0.073 \pm 0.029$ \\
SIGPAF-M & 0.044 & 0.082 & 0.105 & $0.077 \pm 0.031$ \\
SIGPAF-TSK & $\mathbf{0 . 0 4 1}$ & $\mathbf{0 . 0 7 8}$ & 0.101 & $0.074 \pm 0.030$ \\
\hline
\end{tabular}

Table 3. Evaluation of SIGPA, SIGPAF-M, and SIGPAF-TSK on the optimality criteria.

\begin{tabular}{ccccc}
\hline Missions & Objectives & SIGPA & SIGPAF-M & SIGPAF-TSK \\
\hline \multirow{3}{*}{ Mission 1 } & Traveled distance $(\mathrm{km})$ & 2.068 & 2.065 & 2.069 \\
& Path deviations & 3 & 3 & 3 \\
& Energy consumption $(\mathrm{kg} / \mathrm{h})$ & 0.55 & 0.53 & 0.53 \\
Mission 2 & Traveled distance $(\mathrm{km})$ & 3.567 & 3.559 & 3.562 \\
& Path deviations & 6 & 5 & 5 \\
& Energy consumption $(\mathrm{kg} / \mathrm{h})$ & 0.87 & 0.82 & 0.84 \\
Mission 3 & Traveled distance $(\mathrm{km})$ & 4.146 & 4.138 & 4.140 \\
& Path deviations & 10 & 8 & 9 \\
& Energy consumption $(\mathrm{kg} / \mathrm{h})$ & 1.16 & 1.08 & 1.10 \\
\hline
\end{tabular}

Table 4. Experimental results of 10 random generated scenarios of SIGPA, SIGPAF-M, and SIGPAF-TSK.

\begin{tabular}{|c|c|c|c|c|}
\hline Missions & Evaluation Criteria & SIGPA & SIGPAF-M & SIGPAF-TSK \\
\hline & Traveled distance $(\mathrm{km})$ & 3.528 & 3.454 & 3.602 \\
\hline Mission 1 & Path deviations & 7 & 7 & 5 \\
\hline \multirow{3}{*}{ (5 targets, 8 obstacles) } & Energy consumption $(\mathrm{kg} / \mathrm{h})$ & 0.610 & 0.607 & 0.612 \\
\hline & Computing time (ms) & 0.5195 & 0.531 & 0.520 \\
\hline & Traveled distance $(\mathrm{km})$ & 3.663 & 3.594 & 3.594 \\
\hline Mission 2 & Path deviations & 11 & 7 & 9 \\
\hline \multirow[t]{3}{*}{ (7 targets, 1 obstacle) } & Energy consumption $(\mathrm{kg} / \mathrm{h})$ & 0.793 & 0.748 & 0.752 \\
\hline & Computing time (ms) & 0.688 & 0.698 & 0.691 \\
\hline & Traveled distance (km) & 4.308 & 4.335 & 4.294 \\
\hline Mission 3 & Path deviations & 13 & 12 & 13 \\
\hline \multirow[t]{3}{*}{ (9 targets, 10 obstacles) } & Energy consumption $(\mathrm{kg} / \mathrm{h})$ & 0.982 & 0.998 & 0.965 \\
\hline & Computing time (ms) & 0.868 & 0.913 & 0.885 \\
\hline & Traveled distance $(\mathrm{km})$ & 4.496 & 4.428 & 4.450 \\
\hline Mission 4 & Path deviations & 17 & 14 & 14 \\
\hline \multirow{3}{*}{ (12 targets, 4 obstacles) } & Energy consumption $(\mathrm{kg} / \mathrm{h})$ & 1.391 & 1.243 & 1.369 \\
\hline & Computing time (ms) & 1.146 & 1.364 & 1.342 \\
\hline & Traveled distance (km) & 6.557 & 6.279 & 6.356 \\
\hline Mission 5 & Path deviations & 23 & 22 & 23 \\
\hline \multirow{2}{*}{ (13 targets, 6 obstacles) } & Energy consumption $(\mathrm{kg} / \mathrm{h})$ & 1.608 & 1.441 & 1.507 \\
\hline & Computing time (ms) & 1.468 & 1.519 & 1.497 \\
\hline
\end{tabular}


Table 4. Cont.

\begin{tabular}{|c|c|c|c|c|}
\hline Missions & Evaluation Criteria & SIGPA & SIGPAF-M & SIGPAF-TSK \\
\hline & Traveled distance $(\mathrm{km})$ & 6.706 & 6.307 & 6.564 \\
\hline Mission 6 & Path deviations & 18 & 20 & 18 \\
\hline \multirow[t]{3}{*}{ (17 targets, 3 obstacles) } & Energy consumption $(\mathrm{kg} / \mathrm{h})$ & 1.786 & 1.678 & 1.744 \\
\hline & Computing time (ms) & 1.928 & 2.215 & 2.052 \\
\hline & Traveled distance $(\mathrm{km})$ & 9.007 & 8.698 & 8.864 \\
\hline Mission 7 & Path deviations & 26 & 22 & 23 \\
\hline \multirow[t]{3}{*}{ (19 targets, 4 obstacles) } & Energy consumption $(\mathrm{kg} / \mathrm{h})$ & 2.058 & 1.859 & 1.914 \\
\hline & Computing time (ms) & 2.494 & 2.710 & 2.561 \\
\hline & Traveled distance $(\mathrm{km})$ & 9.411 & 8.448 & 8.332 \\
\hline Mission 8 & Path deviations & 29 & 24 & 23 \\
\hline \multirow[t]{3}{*}{ (21 targets, 8 obstacles) } & Energy consumption $(\mathrm{kg} / \mathrm{h})$ & 2.455 & 2.174 & 2.134 \\
\hline & Computing time (ms) & 2.915 & 3.360 & 3.105 \\
\hline & Traveled distance $(\mathrm{km})$ & 10.730 & 9.182 & 9.355 \\
\hline Mission 9 & Path deviations & 32 & 26 & 26 \\
\hline \multirow[t]{3}{*}{ (23 targets, 5 obstacles) } & Energy consumption $(\mathrm{kg} / \mathrm{h})$ & 2.889 & 2.379 & 2.360 \\
\hline & Computing time (ms) & 3.230 & 3.373 & 3.543 \\
\hline & Traveled distance $(\mathrm{km})$ & 11.533 & 10.812 & 10.964 \\
\hline \multirow{3}{*}{$\begin{array}{c}\text { Mission } 10 \\
\text { (25 targets, } 2 \text { obstacles) }\end{array}$} & Path deviations & 36 & 27 & 29 \\
\hline & Energy consumption $(\mathrm{kg} / \mathrm{h})$ & 3.480 & 2.939 & 3.009 \\
\hline & Computing time (ms) & 3.637 & 3.968 & 3.792 \\
\hline
\end{tabular}

\section{Discussion}

In this study, three experiments were conducted to compare the effectiveness of SIGPA, SIGPAF-M, and SIGPAF-TSK algorithms. Overall, the integration of fuzzy logic enhanced the performance of SIGPA algorithm in terms of path optimality with respect to the objectives. However, SIGPA presented a comparable computation time for calculating an optimal path among various POIs. Table 2 shows the mean computation time needed for each algorithm to find an optimal path, while Table 3 shows the quality of the generated paths of each algorithm per mission with respect to the objectives (traveled distance, energy consumption, and path deviations). It is shown that the SIGPA algorithm, due to its evaluation criterion that is based on Mean Square Error, needs less computation time for finding a solution, as the number of the POIs to be visited, or else, the complexity of the problem increases. However, the SIGPAF-TSK presented a comparable performance since the output generation process from the fuzzy inputs is based on weighted average, in contrast with SIGPAF-M where the defuzzification process is followed [44].

Regarding quality of the generated paths, SIGPAF-M succeeded in finding better paths with respect to the objective terms (traveled distance, energy consumption, and path deviations) that are presented in Section 2.1 in all cases. The initial results were also validated by the second loop of experiments, where 10 randomly generated scenarios of increasing complexity (from 5 to 25 targets) and a larger graph of 160 nodes were implemented. These experiments included randomly assigned current velocities and directions to each arc; some nodes were also considered as objects and, thus, were avoided by excluding them from the graph. The results, presented in Table 4, showed that for smaller path planning problems, the SIGPA algorithm reaches comparative performance to SIGPAF approaches. However, as the path planning problem becomes more complicated and more targets should be visited, the fuzzy logic approach seems more suitable. It is notable that SIGPA remains a more computationally efficient approach due to the simplicity of the RMSE evaluation of the objective terms compared with fuzzy approaches. On the other hand, SIGPAF-TSK presented a comparable performance, reaching a better trade-off between computation time and solution quality as the problem's complexity increases. These results are aligned with the literature regarding the differences in operation of Mamdani and Takagi-Sugeno-Kang fuzzy controls. 
The output from Mamdani FIS can be presented linguistically or with a crisp value since the inference result before defuzzification is a fuzzy set [36]. On the other hand, the Sugeno FIS gives a crisp output value obtained by a weighted average of the rules' consequent [37]. Accordingly, these two types of FISs are, in theory, suitable for real-time applications of USV path planning. Based on the advantages of Mamdani FIS, SIGPAF-M has (i) expressive power and interpretable rule consequents; (ii) intuitive and interpretable nature of the rules, which results to the wide use on decision support systems; (iii) the ability to be applied to Multiple-Input Single-Output and Multiple-Input Multiple-Output systems. However, it is less flexible in system design and needs more computational effort compared with SIGPAF-TSK. SIGPAF-TSK can be further optimized with the use of specific algorithms, such as calibrating the weights. Also, SIGPAF-TSK has lower processing time compared with SIGPAF-M due to the replacement of defuzzification process by the calculation of the weighted average. Another advantage of SIGPAF-TSK is its robustness in the case of noise presence, which constitutes greater suitability for processing sensor data. Last, it is more flexibility in the design. However, it can be used only for Multiple-Input Single-Output systems and is not as suitable as Mamdani FIS for decision making due to the lack of a defuzzification process, which results a loss of interpretability [44,45]. However, both approaches presented a comparable performance enhancing the effectiveness of SIGPA algorithm in finding optimal paths for multi-objective graph-based problems.

\section{Conclusions}

This study aims to enhance the performance of SIGPA algorithm by integrating fuzzy logic. To this end, the two most popular FISs (Mamdani and Takagi-Sugeno-Kang) have been evaluated and tested in various experiments for solving the multi-objective graphbased path planning problem in the case of USVs. The results showed that SIGPA remains a credible solution when it comes to computational effort, especially for real time applications, albeit with a loss of solution's quality. On the other hand, Mamdani FIS contributes to the generation of better paths with respect to the objective terms; however, it needs more computation time. To this end, for a balanced solution among computation time and solution quality, the Takagi-Sugeno-Kang can be used $[45,46]$. Therefore, depending on the application and the needs of user, the more-suitable algorithm can be employed.

Future work includes the development of real case studies under various scenarios and conditions by modifying the mathematical modeling to fit to more complex and real conditions.

Author Contributions: Conceptualization, C.N.; methodology, C.N.; software, C.N.; validation, C.N.; formal analysis, C.N.; investigation, C.N.; writing—original draft preparation, C.N.; writingreview and editing, C.N. and D.V.L.; visualization, C.N.; supervision, C.N.; project administration, D.V.L.; funding acquisition, D.V.L. All authors have read and agreed to the published version of the manuscript.

Funding: This research received no external funding.

Institutional Review Board Statement: Not applicable.

Informed Consent Statement: Not applicable.

Acknowledgments: Part of this work is based on the results of the successfully completed three-year research project OPTINET. OPTINET has been co-financed by the European Regional Development Fund of the European Union and Greek national funds through the Operational Program Competitiveness, Entrepreneurship and Innovation, under the call "Research-Create-Innovate" (project code: T1E $\Delta$ K-01907).

Conflicts of Interest: The authors declare no conflict of interest. 


\section{References}

1. Mac, T.T.; Copot, C.; Tran, D.T.; De Keyser, R. Heuristic Approaches in Robot Path Planning: A Survey. Robot. Auton. Syst. 2016, 86, 13-28. [CrossRef]

2. Iakovidis, D.K.; Diamantis, D.; Dimas, G.; Ntakolia, C.; Spyrou, E. Digital enhancement of cultural experience and accessibility for the visually impaired. In Technological Trends in Improved Mobility of the Visually Impaired; Springer: Berlin/Heidelberg, Germany, 2020; pp. 237-271.

3. Ntakolia, C.; Dimas, G.; Iakovidis, D.K. User-Centered System Design for Assisted Navigation of Visually Impaired Individuals in Outdoor Cultural Environments. Univ. Access Inform. Soc. 2020. [CrossRef]

4. Mirjalili, S.; Song Dong, J.; Lewis, A. Ant Colony Optimizer: Theory, Literature Review, and Application in AUV Path Planning. In Nature-Inspired Optimizers: Theories, Literature Reviews and Applications; Mirjalili, S., Song Dong, J., Lewis, A., Eds.; Studies in Computational Intelligence; Springer International Publishing: Cham, Switzerland, 2020; pp. 7-21. ISBN 978-3-030-12127-3.

5. Wang, H.; Guo, F.; Yao, H.; He, S.; Xu, X. Collision Avoidance Planning Method of USV Based on Improved Ant Colony Optimization Algorithm. IEEE Access 2019, 7, 52964-52975. [CrossRef]

6. Singh, Y.; Sharma, S.; Sutton, R.; Hatton, D. Optimal Path Planning of an Unmanned Surface Vehicle in a Real- Time Marine Environment using a Dijkstra Algorithm. In Marine Navigation; CRC Press: Boca Raton, FL, USA, 2017; ISBN 978-1-315-09913-2.

7. Singh, Y.; Sharma, S.; Sutton, R.; Hatton, D.; Khan, A. A Constrained A* Approach towards Optimal Path Planning for an Unmanned Surface Vehicle in a Maritime Environment Containing Dynamic Obstacles and Ocean Currents. Ocean Eng. 2018, 169, 187-201. [CrossRef]

8. Song, R.; Liu, Y.; Bucknall, R. Smoothed A* Algorithm for Practical Unmanned Surface Vehicle Path Planning. Appl. Ocean Res. 2019, 83, 9-20. [CrossRef]

9. Jorge, V.A.M.; Granada, R.; Maidana, R.G.; Jurak, D.A.; Heck, G.; Negreiros, A.P.F.; dos Santos, D.H.; Gonçalves, L.M.G.; Amory, A.M. A Survey on Unmanned Surface Vehicles for Disaster Robotics: Main Challenges and Directions. Sensors 2019, 19, 702. [CrossRef] [PubMed]

10. Ntakolia, C.; Iakovidis, D.K. A Route Planning Framework for Smart Wearable Assistive Navigation Systems. SN Appl. Sci. 2021, 3, 104. [CrossRef]

11. Ntakolia, C.; Iakovidis, D.K. A Swarm Intelligence Graph-Based Pathfinding Algorithm (SIGPA) for Multi-Objective Route Planning. Comput. Oper. Res. 2021, 133, 105358. [CrossRef]

12. Deb, K. Multi-objective Optimisation Using Evolutionary Algorithms: An Introduction. In Multi-Objective Evolutionary Optimisation for Product Design and Manufacturing; Wang, L., Ng, A.H.C., Deb, K., Eds.; Springer: London, UK, 2011; pp. 3-34. ISBN 978-0-85729-652-8.

13. Shen, Y.; Ge, G. Multi-Objective Particle Swarm Optimization Based on Fuzzy Optimality. IEEE Access 2019, 7, 101513-101526. [CrossRef]

14. Davoodi, M.; Panahi, F.; Mohades, A.; Hashemi, S.N. Multi-Objective Path Planning in Discrete Space. Appl. Soft Comput. 2013, 13, 709-720. [CrossRef]

15. Xiang, X.; Yu, C.; Lapierre, L.; Zhang, J.; Zhang, Q. Survey on Fuzzy-Logic-Based Guidance and Control of Marine Surface Vehicles and Underwater Vehicles. Int. J. Fuzzy Syst. 2018, 20, 572-586. [CrossRef]

16. Panda, M.; Das, B.; Subudhi, B.; Pati, B.B. A Comprehensive Review of Path Planning Algorithms for Autonomous Underwater Vehicles. Int. J. Autom. Comput. 2020, 17, 321-352. [CrossRef]

17. Zhao, Y.; Zheng, Z.; Liu, Y. Survey on Computational-Intelligence-Based UAV Path Planning. Knowl.-Based Syst. 2018, 158, 54-64. [CrossRef]

18. Salleh, M.N.M.; Talpur, N.; Hussain, K. Adaptive Neuro-Fuzzy Inference System: Overview, Strengths, Limitations, and Solutions. In Proceedings of the Data Mining and Big Data; Tan, Y., Takagi, H., Shi, Y., Eds.; Springer International Publishing: Cham, Switzerland, 2017; pp. 527-535.

19. Deng, H.; Zhu, J. Optimal Path Planning for Unmanned Vehicles Using Improved Ant Colony Optimization Algorithm. In Proceedings of the Neural Computing for Advanced Applications; Zhang, H., Yang, Z., Zhang, Z., Wu, Z., Hao, T., Eds.; Springer: Singapore, 2021; pp. 701-714.

20. Xin, J.; Zhong, J.; Yang, F.; Cui, Y.; Sheng, J. An Improved Genetic Algorithm for Path-Planning of Unmanned Surface Vehicle. Sensors 2019, 19, 2640. [CrossRef] [PubMed]

21. Xia, G.; Sun, X.; Xia, X. Multiple Task Assignment and Path Planning of a Multiple Unmanned Surface Vehicles System Based on Improved Self-Organizing Mapping and Improved Genetic Algorithm. J. Mar. Sci. Eng. 2021, 9, 556. [CrossRef]

22. Zhang, J.; Zhang, F.; Liu, Z.; Li, Y. Efficient Path Planning Method of USV for Intelligent Target Search. J. Geovis. Spat. Anal. 2019, 3, 13. [CrossRef]

23. Sang, H.; You, Y.; Sun, X.; Zhou, Y.; Liu, F. The Hybrid Path Planning Algorithm Based on Improved A* and Artificial Potential Field for Unmanned Surface Vehicle Formations. Ocean Eng. 2021, 223, 108709. [CrossRef]

24. Zhang, Z.; Wu, D.; Gu, J.; Li, F. A Path-Planning Strategy for Unmanned Surface Vehicles Based on an Adaptive Hybrid Dynamic Stepsize and Target Attractive Force-RRT Algorithm. J. Mar. Sci. Eng. 2019, 7, 132. [CrossRef]

25. Xin, J.; Zhong, J.; Li, S.; Sheng, J.; Cui, Y. Greedy Mechanism Based Particle Swarm Optimization for Path Planning Problem of an Unmanned Surface Vehicle. Sensors 2019, 19, 4620. [CrossRef] 
26. Zhong, J.; Li, B.; Li, S.; Yang, F.; Li, P.; Cui, Y. Particle Swarm Optimization with Orientation Angle-Based Grouping for Practical Unmanned Surface Vehicle Path Planning. Appl. Ocean Res. 2021, 111, 102658. [CrossRef]

27. Wang, N.; Jin, X.; Er, M.J. A Multilayer Path Planner for a USV under Complex Marine Environments. Ocean Eng. 2019, 184, 1-10. [CrossRef]

28. Wang, N.; Xu, H.; Li, C.; Yin, J. Hierarchical Path Planning of Unmanned Surface Vehicles: A Fuzzy Artificial Potential Field Approach. Int. J. Fuzzy Syst. 2020, 23, 1797-1808. [CrossRef]

29. Yu, K.; Liang, X.; Li, M.; Chen, Z.; Yao, Y.; Li, X.; Zhao, Z.; Teng, Y. USV Path Planning Method with Velocity Variation and Global Optimisation Based on AIS Service Platform. Ocean Eng. 2021, 236, 109560. [CrossRef]

30. Ma, Y.; Hu, M.; Yan, X. Multi-Objective Path Planning for Unmanned Surface Vehicle with Currents Effects. ISA Trans. 2018, 75, 137-156. [CrossRef] [PubMed]

31. Xia, G.; Han, Z.; Zhao, B.; Liu, C.; Wang, X. Global Path Planning for Unmanned Surface Vehicle Based on Improved Quantum Ant Colony Algorithm. Math. Probl. Eng. 2019, 2019, 2902170. [CrossRef]

32. Xia, G.; Han, Z.; Zhao, B.; Wang, X. Local Path Planning for Unmanned Surface Vehicle Collision Avoidance Based on Modified Quantum Particle Swarm Optimization. Complexity 2020, 2020, 3095426. [CrossRef]

33. Guo, X.; Ji, M.; Zhao, Z.; Wen, D.; Zhang, W. Global Path Planning and Multi-Objective Path Control for Unmanned Surface Vehicle Based on Modified Particle Swarm Optimization (PSO) Algorithm. Ocean Eng. 2020, 216, 107693. [CrossRef]

34. Li, D.; Wang, P.; Du, L. Path Planning Technologies for Autonomous Underwater Vehicles-A Review. IEEE Access 2019, 7 , 9745-9768. [CrossRef]

35. Zhang, H.; Lin, W.; Chen, A. Path Planning for the Mobile Robot: A Review. Symmetry 2018, 10, 450. [CrossRef]

36. Mamdani, E.H.; Assilian, S. An Experiment in Linguistic Synthesis with a Fuzzy Logic Controller. Int. J. Man-Mach. Stud. 1975, 7, 1-13. [CrossRef]

37. Jang, J.S.R.; Sun, C.T.; Mizutani, E. Neuro-Fuzzy and Soft Computing-A Computational Approach to Learning and Machine Intelligence [Book Review]. IEEE Trans. Autom. Control 1997, 42, 1482-1484. [CrossRef]

38. Chen, Z.; Zhang, Y.; Zhang, Y.; Nie, Y.; Tang, J.; Zhu, S. A Hybrid Path Planning Algorithm for Unmanned Surface Vehicles in Complex Environment With Dynamic Obstacles. IEEE Access 2019, 7, 126439-126449. [CrossRef]

39. Song, R.; Liu, Y.; Bucknall, R. A Multi-Layered Fast Marching Method for Unmanned Surface Vehicle Path Planning in a Time-Variant Maritime Environment. Ocean Eng. 2017, 129, 301-317. [CrossRef]

40. Zeng, W.; Church, R.L. Finding Shortest Paths on Real Road Networks: The Case for A*. Int. J. Geogr. Inf. Sci. 2009, 23, 531-543. [CrossRef]

41. Lerner, J.; Wagner, D.; Zweig, K. (Eds.) Algorithmics of Large and Complex. Networks: Design, Analysis, and Simulation; Theoretical Computer Science and General Issues, Lect. Notes ComputerState-of-the-Art Surveys; Springer: Berlin/Heidelberg, Germany, 2009; ISBN 978-3-642-02093-3.

42. Liu, X.; Li, Y.; Zhang, J.; Zheng, J.; Yang, C. Self-Adaptive Dynamic Obstacle Avoidance and Path Planning for USV Under Complex Maritime Environment. IEEE Access 2019, 7, 114945-114954. [CrossRef]

43. Yan, Z.; Li, J.; Wu, Y.; Zhang, G. A Real-Time Path Planning Algorithm for AUV in Unknown Underwater Environment Based on Combining PSO and Waypoint Guidance. Sensors 2018, 19, 20. [CrossRef] [PubMed]

44. Hamam, A.; Georganas, N.D. A Comparison of Mamdani and Sugeno Fuzzy Inference Systems for Evaluating the Quality of Experience of Hapto-Audio-Visual Applications. In Proceedings of the 2008 IEEE International Workshop on Haptic Audio Visual Environments and Games, Ottawa, ON, Canada, 18-19 October 2008; pp. 87-92.

45. Wang, Y.; Chen, Y. A Comparison of Mamdani and Sugeno Fuzzy Inference Systems for Traffic Flow Prediction. J. Comput. 2014, 9, 12-21. [CrossRef]

46. Davis, D.; Supriya, P. Implementation of Fuzzy-Based Robotic Path Planning. In Proceedings of the Second International Conference on Computer and Communication Technologies; Satapathy, S.C., Raju, K.S., Mandal, J.K., Bhateja, V., Eds.; Springer India: New Delhi, India, 2016; pp. 375-383. 